\author{
S.C. $\mathrm{Ng}^{1}$, N. Ismail ${ }^{1,2}$, Aidy Ali ${ }^{1}$, Barkawi Sahari ${ }^{1}$, J.M. Yusof ${ }^{2}$ \\ ${ }^{1}$ Intelligent System and Robotic Laboratory, Institute of Advanced Technology, Universiti \\ Putra Malaysia, 43400 Serdang, Selangor, Malaysia,ngsokchoo@gmail.com \\ ${ }^{2}$ Department of Mechanical and Manufacturing Engineering, Faculty of Engineering, \\ Universiti Putra Malaysia, 43400 Serdang, Selangor, Malaysia
}

\title{
EXPERIMENTAL INVESTIGATION ON EFFECTIVE DETECTION OF DELAMINATION IN GFRP COMPOSITES USING TAGUCHI METHOD
}

\begin{abstract}
Detection of delamination defect in glass fiber reinforced plastics (GFRP) by using ultrasonic testing has been a challenging task in industry. The properties of the constituent materials, fiber orientation and the stacking sequence of laminated composite materials could cause high attenuation of ultrasound signals. Ultrasonic testing is based on the interpretation of the reflected ultrasound signals when a transducer imposes ultrasound waves (pulse) to a material. It is difficult to differentiate if the reflected signal is induced from the defects, fiber content or the intermediate layers of GFRP composites. Most of the time, the drastic attenuation of signals could enshroud the modest changes in the reflected signals from defects. The purpose of this paper is to investigate the influence of fiber orientation, thickness and delamination of GFRP composites on the rise time, pulse duration and attenuation ratio of the reflected ultrasound signal. The rise time, pulse duration and attenuation ratio of Ascan data was observed with respect to different positions of damage (delamination), thickness and stacking sequence of the lamina. It is essential to identify the significant factors that contribute to the abnormal characteristics of the reflected signals in which the defect is identified. Moreover, this paper presents the application of Taguchi method for maximizing the detection of defect in GFRP composites influenced by delamination. The optimum combination of the significant contributing factor for the signal's abnormal characteristics and its effect on damage detection was obtained by using the analysis of signal-to-noise ratio. The finding of this study revealed that delamination is the most influential factor on the attenuation ratio.
\end{abstract}

Key words: glass fiber reinforced plastics, ultrasonic testing, attenuation, delamination

\section{INTRODUCTION}

Glass fiber reinforced plastics (GFRP) composites have wide application in manufacturing and advanced industry such as aircraft, vessels and tanks due to their high tensile and compressive strength, corrosion resistance, light weight construction and resistance to chemical and microbiological attack. However, multilayered composite structures are susceptible to mechanical loading and temperature degradation [1]. Typical failure modes in laminated composites are delamination, matrix cracks and fiber breakage [2][3]. The defect that is most commonly found in composites is delamination that occurs parallel to surface of the structure [4]. This phenomenon can be caused by several factors: (1) air that is trapped between prepreg sheets in the lay up process, (2) failure of carefully removing the prepreg 
backing film during manufacturing, and (3) external loads during in-service. These failure modes affect the mechanical properties of the composites and hence degrade the performance of the composite structures. The growth of delamination at the internal part of a structure is beyond human visibility until severe damages propagate to the surface of the structure that could cause unbearable loss to industry. Thus, non-invasive damage evaluations are crucial to inspect the failure in composite materials for the purpose of ensuring the integrity of a structure.

Over the last decades, ultrasonic techniques have been the most widely used non-destructive testing and evaluation (NDE\&T) method to analyze the defects occurred inside a structure [57]. Through-transmission or pulse-echo techniques are applied in which the specimen under test is induced by ultrasonic pulses and the transmitted or reflected pulse is interpreted. By taking into account the maximal amplitude of ultrasonic echoes to compute its rise time, pulse duration and fall time, the nature, size and orientation of defects in a material can be characterized. However, the efficient detection and characterization of delaminations occurring in multilayered composite plates still remains challenge for researchers [8-9]. This could be due to two major factors such that the fiber orientation and stacking sequence of the composite lamina could probably cause the high attenuation of the reflected signals. The scattering effect caused by the composite's coarse-grained structure may annihilate the return echoes and obscure the defect visibility. In general, the pulse width is significantly larger than the gap distance of delaminations [10]. Consequently, the resolution in the material's thickness direction is poor. The stacking sequence and fiber orientation of lamina could be the factors of delaying the rise time of the pulse instead of the internal defects. The purpose of this paper is to investigate the influence of fiber orientation, material's thickness and delamination of GFRP composites on the pattern of the reflected ultrasound signal in which the defect is identified. This can be determined through a series of experiments. Conventional experimental design method such as factorial design is complex with the increasing number of parameters [11]. Moreover, the conventional design method fails to consider any possible interactions between the parameters [12]. In manufacturing process, it is unlikely to study all the factors involved in the process for the sake of determining their main effect in a single experiment. Thus, the design of experiment at most of the time does not reach the desired objectives [13]. Random design on the other hand is applicable for the large scale system that involves many parameters but with few interactions occur between parameters. Taguchi method has been used in enormous amount of research to optimize process parameters with minimum of experimental runs [12]. In this study, Taguchi method design was applied to identify the significant contributing factors that affect the behaviour of the received ultrasound signals in NDE\&T ultrasonic testing on GFRP composite. The influence of the factor to the variability of the result is then measured by signal-to-noise ratio analysis.

\section{MATERIALS AND EXPERIMENTAL TECHNIQUE}

\section{Materials}

Two sets of GFRP composites with different lay-ups $\left(\left[0^{\circ}\right]_{4},\left[90^{\circ}\right]_{4},\left[+45^{\circ} / 0^{\circ} / 45^{\circ} / 90^{\circ}\right]\right.$ and random orientation) manufactured by Hexagon Inc. (Malaysia) was chosen as test materials. The GFRP composed of E-glass fibers and epoxy resin. Two sets of the GFRP composites were approximately $10.0 \mathrm{~mm}$ and $3.0 \mathrm{~mm}$ of thickness respectively to denote the thick and thin 
composites. The test material specimen has the area of $200 \mathrm{~mm}$ x $200 \mathrm{~mm}$. Delamination was introduced by inserting a Mylar sheet between the lamina. There were three combinations of position that the Mylar sheet was inserted in the test materials: front $(f)$, middle $(m)$ and bottom (b). For test material $f$, the Mylar sheet was located between the fist layer (side 2) and second layer of the GFRP. For test material $m$, the Mylar sheet was located at the middle location of the GFRP. For test material $b$, the Mylar sheet was located between the second last layer (side 2) and the last layer of the GFRP. The detailed dimension and structure of the test material are depicted in Fig. 1.
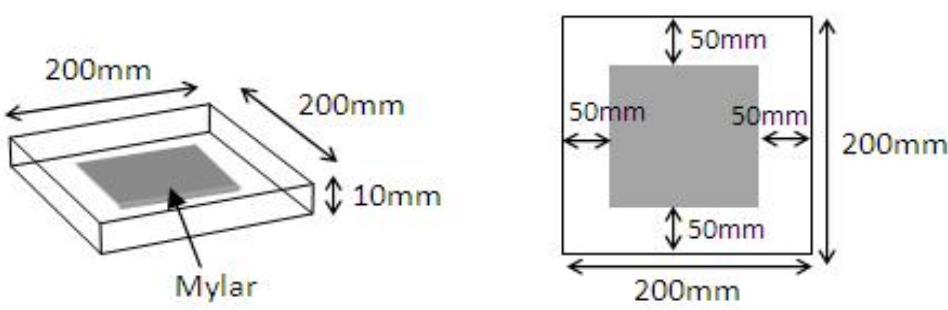

Fig. 1. Dimension of GFRP

\section{Hardware set up}

A single transducer with $1 \mathrm{MHz}$ centre frequency and diameter of $31.24 \mathrm{~mm}$ acted as transmitter and receiver of ultrasonic signals (Fig. 2). The ultrasonic testing system worked as follows [1]: The transducer, working on piezoelectric effect [14], transformed electrical pulses into mechanical vibrations to generate ultrasonic waves (pulse). The transducer was placed onto the GFRP specimen and the pulse generated was transmitted into the test object (GFRP). The pulse travelled through the test object and responded to the object's a boundary (i.e. voids, cracks or delamination). The returned signal (echo) was then transformed back into electrical pulses that can be observed on an oscilloscope.
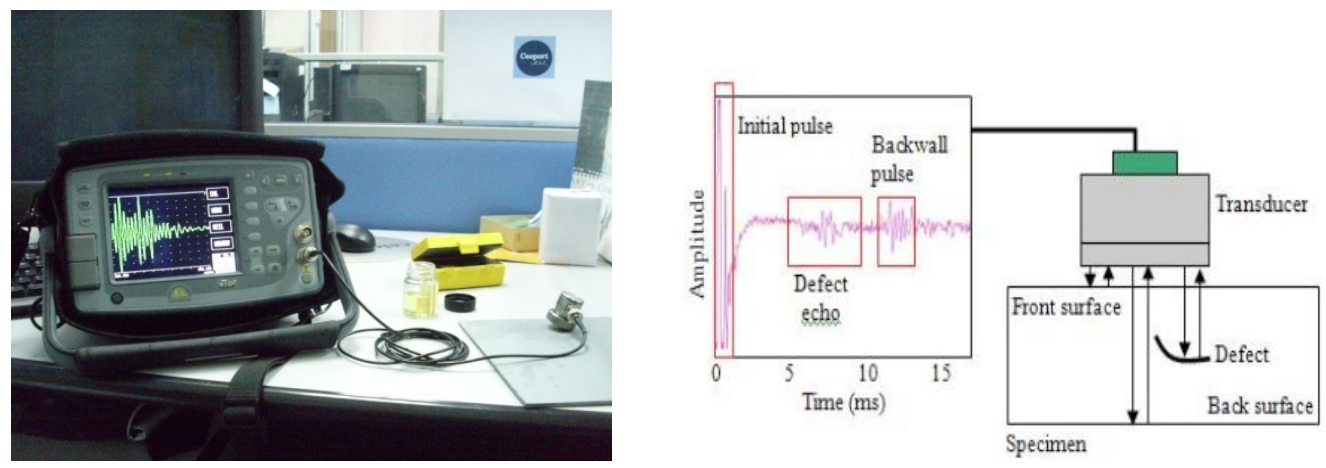

Fig. 2. Experimental setup of pulse echo ultrasonic testing

The depth of the defect ( $d$ ) of the test object from the surface is measured by Eq. (1):

$$
d=\frac{v T_{\text {tof }}}{2}
$$


where $v$ is the velocity of ultrasonic wave in the material and $T_{\text {tof }}$ is time of flight (TOF) measurement of the reflected echo. Fig. 3 shows the response of the damaged specimen. The time for the maximum peak of two successive pulses for the specimen was captured. The presence of defects in the damaged specimen causes the delay of the pulse and thus increases the value of TOF. A $2 \mathrm{kS} / \mathrm{s}$ sampling rate was used to acquire the signals from the receiver.
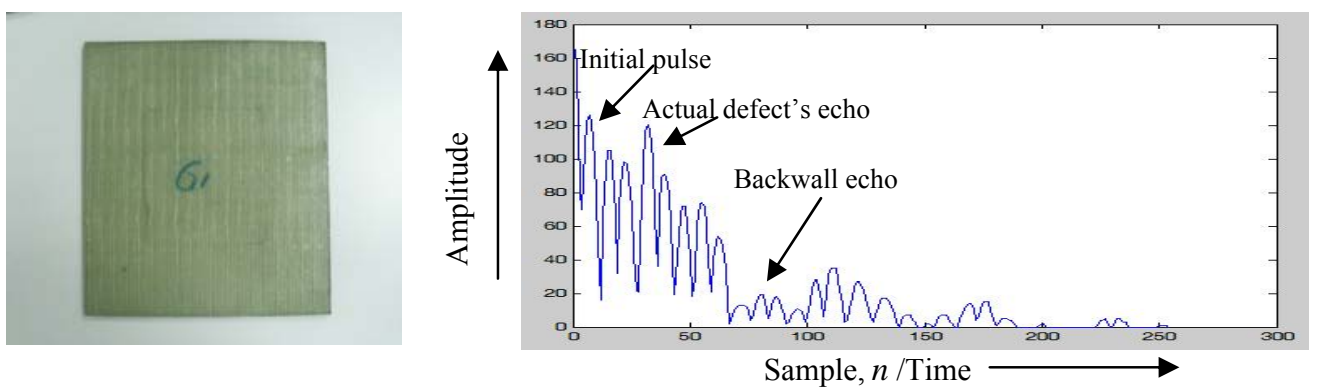

Fig. 3. GFRP specimen with Mylar induced delamination (b) Response of damaged specimen

\section{Taguchi experimental design approach}

The present investigation uses Taguchi method to design the experiment to identify the significant contributing factors on the response of ultrasonic signals in GFRP materials. This method overcomes the drawback of conventional experimentations that involve one factor at a time in the experimental process. In the conventional experimental approach, the number of experiments increases relatively to the number of process parameters. Taguchi method provides an efficient and systematic approach to estimate the contribution of each individual factor and their interactions in the process response. The variation in a process response can be caused by three types of factors [15]: controllable factors, signal factors and noise factors. Controllable factors have an influence on the variability of the process response and these factors can be adjusted during the experiments. The signal factors influence the average value of the response and thus these factors do not pose significant impacts on the variance in the process response. The noise factors have an influence on the process response but these factors are uncontrollable or it is costly to do so. All these factors act upon the process in varying intensities. Therefore, a process study has to be carried out by observing the changes in response when the controllable factors are modified in order to determine signal factor levels, taking into consideration the variability that the noise produce. Taguchi method uses a special design of orthogonal arrays to study the entire parameter space with small number of experiments [16]. The step of Taguchi experimental design are [12]: (1) to identify the response function and the process variables (2) to determine the number of levels for the process parameters (3) to select the appropriate orthogonal array (4) to select the optimum level of process parameters through signal-to-noise ratio $(\mathrm{S} / \mathrm{N})$ analysis and $(5)$ to perform confirmatory experiments if it is necessary. In the present study, three control factors were considered: stacking sequence of the lamina (F), GFRP thickness (T) and the location of Mylar insertion that denotes the position of the damage (D). There are four, two and three levels for each of the factors respectively (Table 1). For thickness factor, GFRP with the thickness of $3.0 \mathrm{~mm}$ and $10.0 \mathrm{~mm}$ were chosen as thin and thick composites respectively. Levels of front, middle and bottom were chosen for the location of Mylar insertion factor. Front denoted that the Mylar was located between the second last and the last layer of the 
lamina. Middle denoted that the Mylar was located at the middle layer of the GFRP. Bottom denoted that the Mylar was located between the first layer (side 2) and second layer of the GFRP. The experimental design was according to an L16 orthogonal array with three columns and sixteen rows (Table 2). Therefore, sixteen experiments are required to study the entire process.

Table 1. Control factors and their respective levels

\begin{tabular}{|l|l|l|l|l|}
\hline Factor & Level 1 & Level 2 & Level 3 & Level 4 \\
\hline $\begin{array}{l}\text { Stacking sequence of } \\
\text { lamina (F) }\end{array}$ & {$\left[0^{\circ}\right]_{4}$} & {$\left[90^{\circ}\right]_{4}$} & {$\left[+45^{\circ} / 0^{\circ} /-45^{\circ} / 90^{\circ}\right]$} & Random \\
\hline GFRP thickness (T) & $3.0 \mathrm{~mm}$ & $10.0 \mathrm{~mm}$ & & \\
\hline Location of Mylar (D) & Front & Middle & Bottom & \\
\hline
\end{tabular}

Table 2. Orthogonal array of Taguchi $L 16$

\begin{tabular}{|l|l|l|l|}
\hline Experiment & $\begin{array}{l}\text { Stacking sequence } \\
\text { of lamina (F) }\end{array}$ & GFRP thickness (T) & $\begin{array}{l}\text { Position of damage } \\
(\mathbf{D})\end{array}$ \\
\hline 1 & 1 & 1 & 1 \\
\hline 2 & 1 & 2 & 2 \\
\hline 3 & 1 & 1 & 3 \\
\hline 4 & 1 & 1 & 4 \\
\hline 5 & 2 & 2 & 1 \\
\hline 6 & 2 & 1 & 2 \\
\hline 7 & 2 & 2 & 3 \\
\hline 8 & 2 & 1 & 4 \\
\hline 9 & 3 & 1 & 1 \\
\hline 10 & 3 & 2 & 2 \\
\hline 11 & 3 & 1 & 3 \\
\hline 12 & 3 & 2 & 4 \\
\hline 13 & 4 & 2 & 1 \\
\hline 14 & 4 & 1 & 2 \\
\hline 15 & 4 & 2 & 3 \\
\hline 16 & 4 & 1 & 4 \\
\hline
\end{tabular}

Taguchi analysis was performed by using signal-to-noise ratio analysis in which the process design is optimized with the reduced variability of the process against uncontrollable factors or noise factors. In this study, 'higher is the best' function (Eq.2) was applied meaning that a high value of signal-to-noise ratio implies the significant influence of controllable factors to the process response [11].

$$
\frac{S}{N}=-\log \frac{1}{n}\left(\sum \frac{1}{y^{2}}\right)
$$

where $n$ is the number of observations in each of the sixteen trials and $y$ is the observed data from the $n$ number of observations for each trial. From signal-to-noise ratio analysis, it can be observed that the process response reacts differently upon different levels of a signal factor instead of the experimental noise. 


\section{RESULTS AND DISCUSSION}

The relative importance of the stacking sequence of lamina, GFRP thickness and position of damage in relation to the receiving signal's rise time, pulse duration and attenuation ratio was investigated. Attenuation ratio denotes the decay rate of the travelling wave as it propagates through a material. It was examined by the decay of the reflected signals in the ultrasonic testing. In the present study, three runs (observations) were performed for each of the sixteen trials. By applying Eq.2, the values of signal-to-noise ratio of the three controllable factors, F, $\mathrm{T}$ and $\mathrm{D}$, upon the signal's rise time, pulse duration and attenuation ratio were shown in Table 3-5. Fig. 4-6 shows the graph of the main effect of the control factors with different levels to the process response in terms of rise time, pulse duration and attenuation ratio of the receiving signal. The results in Fig. 6 illustrated that the position of the damage (location of Mylar insertion) has the most significant impact on the attenuation ratio of the reflected signal. The reflected signal attenuates $16.6 \%(4.4 \mathrm{db})$ from level 1 to level 3 for the factor of position of the damage. It is due to the reverberation of sound waves against the walls of void (delaminations) that causes the conversion of acoustic to thermal energy [17]. For multilayered structure materials such as GFRP, interlayer wave leakage results in the attenuation loss of ultrasound signals. However, comparing to the factor of position of the damage, the stacking sequence of lamina has less effect on the attenuation ratio in which the difference of signal-to-noise ratio value of the reflected signal between level 2 and level 4 is $3.6 \mathrm{db}$

Table 3. $S / N$ response table of $F, T$ and $D$ factors on signal's rise time

\begin{tabular}{lr|rrr}
\hline Level & \multicolumn{1}{|c}{$\boldsymbol{F}$} & $\boldsymbol{T}$ & $\boldsymbol{D}$ \\
\hline & 1 & 10.22908576 & 10.65049287 & 11.5490923 \\
& 2 & 12.40379003 & 11.31406042 & 9.7579002 \\
& 3 & 14.80931075 & 10.8847408 & \\
& 4 & 7.815777774 & & \\
\multicolumn{1}{l|}{ S $\mathrm{N}$ ratio } & 7 & 0.6 & 1.6 \\
Rank & 1 & 3 & 2 \\
\hline
\end{tabular}

Table 4. S/N response table of $F, T$ and $D$ factors on signal's pulse duration

\begin{tabular}{lr|rrr}
\hline Level & $\boldsymbol{F}$ & $\boldsymbol{T}$ & $\boldsymbol{D}$ \\
\hline & 1 & 13.96258598 & 15.15550786 & 14.10755122 \\
& 2 & 17.95044173 & 15.91566607 & 19.95933009 \\
& 3 & 15.56839361 & 17.45020677 & \\
& 4 & 17.29484022 & & \\
$\Delta$ S/N ratio & 4 & 1.5 & 5.9 \\
Rank & 2 & 3 & 1 \\
\hline
\end{tabular}

Table 5. S/N response table of $F, T$ and $D$ factors on signal's attenuation ratio

\begin{tabular}{lr|rrr}
\multicolumn{1}{l|}{ Level } & $\boldsymbol{F}$ & $\boldsymbol{T}$ & \multicolumn{1}{c}{$\boldsymbol{D}$} \\
\hline & 1 & 24.40838772 & 22.18183394 & 24.14607628 \\
& 2 & 26.02915594 & 23.16675497 & 24.5341164 \\
& 3 & 24.93319968 & 26.58202752 & \\
4 & 22.42313751 & & \\
\multicolumn{1}{l|}{ S/N ratio } & 3.6 & 4.4 & 0.4 \\
\hline
\end{tabular}




\section{\begin{tabular}{l|lll}
\hline Rank & 2 & 1 & 3 \\
\hline
\end{tabular}}

From the results of Fig. 4, it is noted that the thickness of the lamina has the most significant impact on the pulse duration of the ultrasonic signal. Pulse duration denotes the TOF of the ultrasonic signal. The pulse takes longer time to travel back to the receiving sensor when the lamina becomes thicker. On the other hand, the signal's rise time was most affected by the stacking sequence of lamina (Fig. 5) in which surface friction of the lamina due to fiber distribution poses long rise time to the ultrasound signal. It is noted that the thickness of lamina and the stacking sequence of the lamina have obscured the impact of position of the damage on both pulse duration and rise time of the ultrasound signal during the ultrasonic testing. The finding of the experiment shows that the highest value of attenuation ratio was obtained at the lowest position of delamination in the GFRP composites (Table 4).

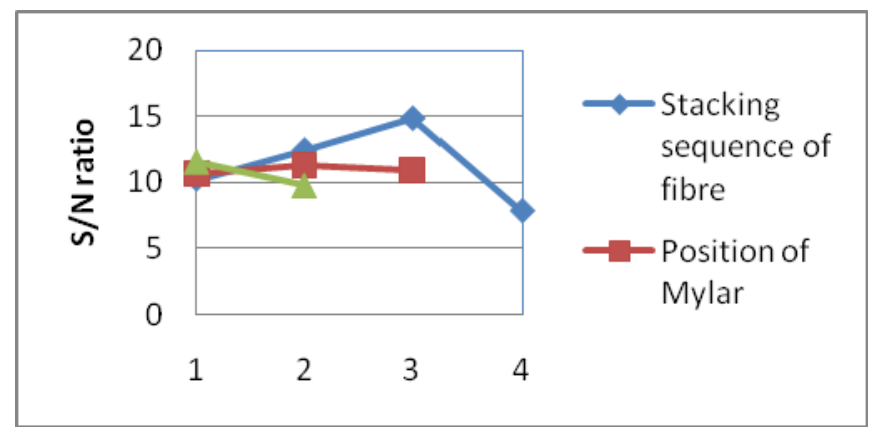

Fig. 4. Main effect of $F, T$ and $D$ factors on signal's rise time

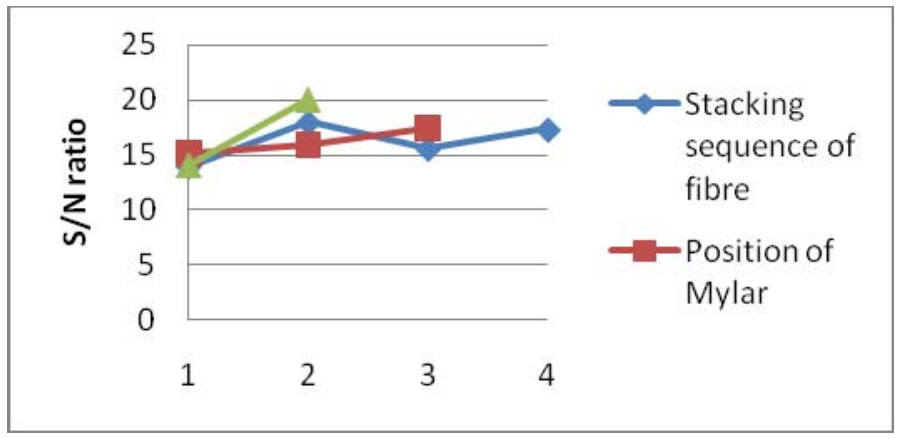

Fig. 5. Main effect of $F, T$ and $D$ factors on signal's pulse duration

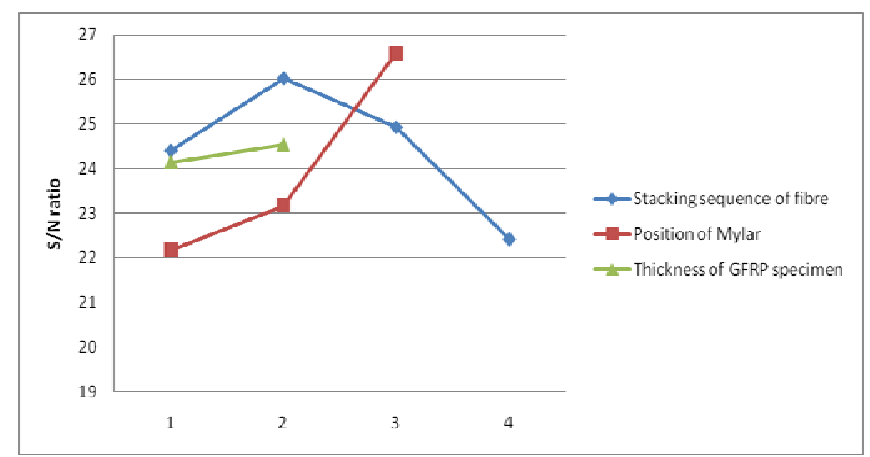

Fig. 6. Main effect of $F, T$ and $D$ factors on signal's attenuation ratio 


\section{CONCLUSIONS}

This paper presented the application of Taguchi experimental design approach to investigate the significant control factor and its relation to the response function in ultrasonic NDE for multi-layered composite materials. Taguchi method was used in this study as this method eliminates the number of experiment and it can demonstrate the interaction between the controllable factors upon the process response. In this study, there were three controllable factors, namely stacking sequence of the lamina, GFRP thickness and the location of Mylar insertion that denotes the position of the damage. The process response towards these factors were ultrasound signal's pulse duration, rise time and attenuation ratio. Ultrasonic testing acting on pulse-echo mode was conducted on GFRP materials to obtain the data. Experimental results showed that the delaminination has the significant influence on attenuation ratio of the ultrasound signal. Although delaminination affects both pulse duration and rise time of the ultrasound signal, its impact was not significant compare to the factors of stacking sequence of the lamina and GFRP thickness. The finding of this study aids the effective detection of delamination defects in multi-layered structure composite materials.

\section{ACKNOWLEDGEMENTS}

This work was supported by the FRGS fund of Malaysia under Grant No. 5523439.

\section{REFERENCES}

1. Ng S.C., Ismail N., Aidy Ali, Barkawi Sahari, Yusof J.M. and Chu B.W.: Non-destructive inspection of multi-layered composite using ultrasonic signal processing. IOP Conference Series: Material Science and Engineering (2011), 17012045.

2. Reifsnider K.: Damage in composite materials. ASTM STP775, American Society for Testing and Materials, Philadelphia, 1982.

3. Ambu, R., Aymerich, F., Ginesu, F. and Priolo, P.: Assessment of NDT interferometric techniques for impact damage detection in composite laminates. Composites Science and Technology 66(2006), 199-205.

4. Adams, R.D. and Cawley, P.: Defect types and non-destructive testing techniques for composites and bonded joints. Constructions and Building Materials 3(1989), 170-183.

5. Brizuela, J., Fritsch, C., and Ibáñez, A.: Railway wheel-flat detection and measurement by ultrasound. Transportation Research Part C: Emerging Technologies 19(2011), 975-984.

6. Ohara Yoshikazu, Horinouchi Satoshi, Hashimoto Makoto, Shintaku Yohei, Yamanaka Kazushi.: Nonlinear ultrasonic imaging method for closed cracks using subtraction of responses at different external loads. Ultrasonics 51(2011), 661-666. 
7. Krishnan Balasubramaniam, Jitendra S. Valluri and Raghu V. Prakash.: Creep damage characterization using a low amplitude nonlinear ultrasonic technique. Materials Characterization, 62(2011), 275-286.

8. Zumpano, G., and Meo, M.: Damage localization using transient non-linear elastic wave spectroscopy on composite structures. International Journal of Non-Linear mechanics, 43(2007), 217-30.

9. Meo, M., Polimeno, U. and Zumpano, G.: Detecting damage in composite material using nonlinear elastic wave spectroscopy methods. Applied Composite Materials 15(2008), 115-126.

10. Wooh, S.C. and Wei, C.A.: High-fidelity ultrasonic pulse-echo scheme for detecting delaminations in composite laminates. Composites Part B: Engineering 30(1999), 433-441.

11. Kilickap, E.: Optimization of cutting parameters on delamination based on Taguchi method during drilling of GFRP composite. Expert Systems with Applications 37(2010), 6116-6122.

12. Davidson, M. Joseph, Balasubramanian, K. and Tagore, G.R.N.: Experimental investigation on flow-forming of AA6061 alloy - a Taguchi approach. Journal of Materials Processing Technology 200(2008), 283-287.

13. Rosa, J.L., Robin, A. M.B. Silva, C.A. Baldan, M.P. Peres.: Electrodeposition of copper on titanium wires: Taguchi experimental design approach. Journal of Materials Processing Technology 209(2009), 1181-1188.

14. Diamanti, K., Soutis, C. and Hodgkinson, J.M.: Lamb waves for the non-destructive inspection of monolithic and sandwich composite beams. Composites Part A: Applied Science and Manufacturing 36(2005), 189-195.

15. Irusta, R., Antolín, G., Velasco, E. and García, J.C.: The selection of testing methods for biofuels using the Taguchi signal-to-noise ratio. Biomass and Bioenergy 6(1994), 405-413.

16. Bagci, E. and Ozcelik, B.: Analysis of temperature changes on the twist drill under different drilling conditions based on Taguchi method during dry drilling of Al 7075-T651. The International Journal of Advanced Manufacturing Technology 29(2006), 629-636. 